\title{
Cytotoxic Effects of Flubendazole in Human Lung Cancer Cell Line
}

\section{A549}

\author{
Elham Hoveizi $\mathbb{G}^{1,{ }^{*}}$ and Fatemeh Fakharzadeh Jahromi ${ }^{1}$ \\ ${ }^{1}$ Department of Biology, Faculty of Science, Shahid Chamran University of Ahvaz, Ahvaz, Iran \\ "Corresponding author: Department of Biology, Faculty of Science, Shahid Chamran University of Ahvaz, Ahvaz, Iran. Email: e.hoveizi@scu.ac.ir
}

Received 2020 August 27; Revised 2020 September 15; Accepted 2020 September 16.

\begin{abstract}
Background: The development of effective anticancer drugs is a significant health issue. Previous studies showed that members of the benzimidazole family have anticancer effects on several cancers

Objectives: The present study investigated the cytotoxic effect of flubendazole on A549 human lung cancer cells.

Methods: The A549 cells were treated with flubendazole at 1, 2, 5, and $10 \mu \mathrm{M}$ concentrations for three days. Cell viability was measured by the MTT assay and Acridine orange staining. Also, the expressions of P62 and Beclin - 1 were analyzed by qRT-PCR analysis.

Results: Cell viability of A549 cells, in a concentration-dependent manner, showed significant differences between the treatment and control groups, and the IC50 value was determined to be $2 \mu \mathrm{M}$. Also, flubendazole reduced the expression of P62 and increased the expression of Beclin 1 in treated cells.

Conclusions: Flubendazole induces cell death in A549 cells in a dose and time-dependent manner and can offer new factors in lung cancer therapeutic strategies.
\end{abstract}

Keywords: Flubendazole, Cytotoxic, Lung Cancer, Cell Death

\section{Background}

Cancer is a global public health problem, and massive expenses are annually spent on research to prevent and treat this disease. Its standard treatment approaches include surgery, radiotherapy, immunotherapy, and chemotherapy $(1,2)$. Among them, chemotherapy has been one of the most effective and potent methods for the treatment of malignant tumors. However, drug resistance is a big obstacle to the successful treatment of cancer by chemotherapy, a mechanism by which cancer cells become resistant to chemical drugs (3).

Cancer rates are increasing day by day, while there is no ideal treatment for cancer yet. Thus, new anticancer drug replacements are required, and there is a continuous effort for new anticancer drug production. Flubendazole is a benzimidazole derivative that has been presented as an anthelminthic drug. The anthelminthic effect of flubendazole is exerted by inhibiting microtubules and blocking glucose uptake that depletes glycogen reserves in larval and adult stages of sensitive parasites and reduces overall ATP formation, causing parasite immobilization $(4,5)$.

Benzimidazole antihelminthics like albendazole, flubendazole, and mebendazole are affirmative medicines for the treatment of major groups of parasitic helminths. These medicines have numerous advantages like safety and capability for anticancer drugs. Also, benzimidazole family members have been approved by the Food and Drug Administration (FDA), and they have been determined by the World Health Organization (WHO) in the list of most important drugs that are essential for the human health system (6). This family shows significant safety and impressive antitumor effects, as potent microtubule disrupting and anti-metastatic agents, immune checkpoint inhibitors, anti-angiogenic agents, and apoptosis increasing agents (7).

Flubendazole induces oxidative stress and DNA damage and leads to apoptotic-like changes in parasites (8). Flubendazole also has anti-proliferative effects on several tumor cell lines and can be an approach for cancer therapy in cancer cells escaping apoptosis signaling by an increase in the production of reactive oxygen species and oxidative stress $(9,10)$. These properties explain the potential of these medicines as inhibitors of cell division and acceptable anticancer agents In chemotherapy. 


\section{Objectives}

As lung cancer is one of the most common cancers in both women and men growing worldwide, this study aimed to evaluate the anti-proliferative and cytotoxic effects of flubendazole on human lung cancer cell line A549. Treatment with benzimidazole family members, like flubendazole, has recently been conducted as potent anticancer agents because of the low cost as generic drugs, long history of safe use in the human population, and easy access. Here, we hypothesized that flubendazole could show cytotoxic effects and induce autophagy in A549 cells.

\section{Methods}

This experimental study was conducted on lung cancer cell line A549 at the Cell and Developmental Lab of the Biology Department, Faculty of Sciences, Shahid Chamran University (ethical code: EE.97.243.93365/scu.ac.ir). The flubendazole dose and treatment time were considered as the study variables. We used six repetitions for each group, while replication was considered as a standard template. Flubendazole solution was prepared at concentrations of $1,2,5$, and $10 \mu \mathrm{M}$ in DMSO and diluted with a medium, then filtered and stored at $4{ }^{\circ} \mathrm{C}(11,12)$.

Cancer cell line A549 was purchased from the Iran Pasteur Institute, Tehran, Iran. Cells were cultured with Dulbecco's modified Eagle's (DMEM, Gibco, USA) medium supplemented with 10\% Fetal Bovine Serum (FBS, Gibco, USA), penicillin $(100 \mathrm{U} / \mathrm{mL})$, and streptomycin $(100 \mu \mathrm{g} / \mathrm{mL})$ in a humidified incubator at $37^{\circ} \mathrm{C}$ with $5 \% \mathrm{CO}_{2}$ and cells with $80 \%$ confluency were passaged. For the observation of cell morphology, the cells were stained by Giemsa in each group, and imaging was performed by inverted microscopy (Labomed, USA). The A549 cancer cells' metabolic activity was measured using the 3-(4,5-dimethylthiazol-2yl)-2,5-diphenyltetrazolium bromide (MTT) reduction assay. Cells were seeded at a density of $1 \times 10^{4}$ cells/well in 96well plates and incubated under standard conditions with the given concentrations of flubendazole for one, two, and three days. Then, $5 \mathrm{mg}$ mL-1 of MTT solution was added to each well, and the plates were incubated at $37^{\circ} \mathrm{C}$ for 4 h. The medium was removed, and the formazan crystals were dissolved in DMSO, and absorbance at $570 \mathrm{~nm}$ was measured using a microplate reader (Expert 96, Asys Hitch, Ec Austria). Also, 1:1 Acridine orange/ethidium bromide stain was prepared for checking apoptosis. Two groups of A549 cells, including the treated group (by IC50 concentration of flubendazole) and the control, were stained after one day of culture in 96-well plates. Images were captured by fluorescent microscopy to determine cell viability (Olympus, Japan). For qRT-PCR analysis, RNXTM (Plus,
Sinagen Company) was used to extract RNA according to its protocol. A nanodrop device (Rush, USA) was used for RNA measurement. The sequence of primers was extracted from the PubMed website (Table 1). In this study, the firststrand cDNA synthesis kit (Takara, Japan), including dNTPs, reverse transcriptase enzyme, primer, and reaction buffer, was utilized to manufacture cDNA. A RUSH device was used to perform the final step of qRT-PCR, and a GAPDH gene was used to normalize each test. The data were analyzed using Armonk, NY, USA, by one-way ANOVA. The curves were drawn by Microsoft Excel (2016), and P values $<0.05$ were considered as significant differences.

\begin{tabular}{lc}
\hline Table 1. Primer Sequence for qRT-PCR & \\
\hline Name & Primer Sequence $\left(\mathbf{5}^{\prime} \rightarrow \mathbf{3}^{\prime}\right)$ \\
\hline GAPDH $(\mathbf{F})$ & GCAAGAGCACAAGAGGAAGA \\
\hline GAPDH $(\mathbf{R})$ & ACTGTGAGGAGGGGAGATTC \\
P62 $(\mathbf{F})$ & CCTGGGTTTCCGTTCACT \\
$\mathbf{P 6 2}(\mathbf{R})$ & TACTTTGGTCCGCTTTCC \\
Beclin-1 (F) & GAGGGATGGAAGGGTCTAAG \\
\hline Beclin-1 $(\mathbf{R})$ & GCCTGGGCTGTGGTAAGT \\
\hline
\end{tabular}

\section{Results}

Morphologic observation showed that flubendazole could induce considerable alterations in A549 cancer cells, like shrinkage, nuclear condensation, cellar rounding, and large vacuoles (Figure 1). The result of the cell viability assay for one-day cell cultures showed significant differences in cell viability between treated cells and control samples. After one day, there was a significant decrease in the viability of A549 cells treated with flubendazole at a given concentration compared to controls ( $\mathrm{P} \leq 0.05$ ). The percentage of viability strongly decreased in treated cells compared to the control group after 1, 2, and 3 days. ( $\mathrm{P} \leq$ 0.05 ) (Figure 2). Acridine orange/ethidium bromide staining was applied to determine the effect of IC50 concentration of flubendazole on A549 cell line morphology and viability. Observations were in line with the MTT results and showed that flubendazole significantly decreased viability and could induce cell death in A549 cells (Figure 3). Also, our results indicated that flubendazole induced cell death (autophagy) initiation by decreasing P62 and increasing Beclin 1 as autophagy markers. The gene expression changes were significantly different between treated cells and controls (Figure 4). 

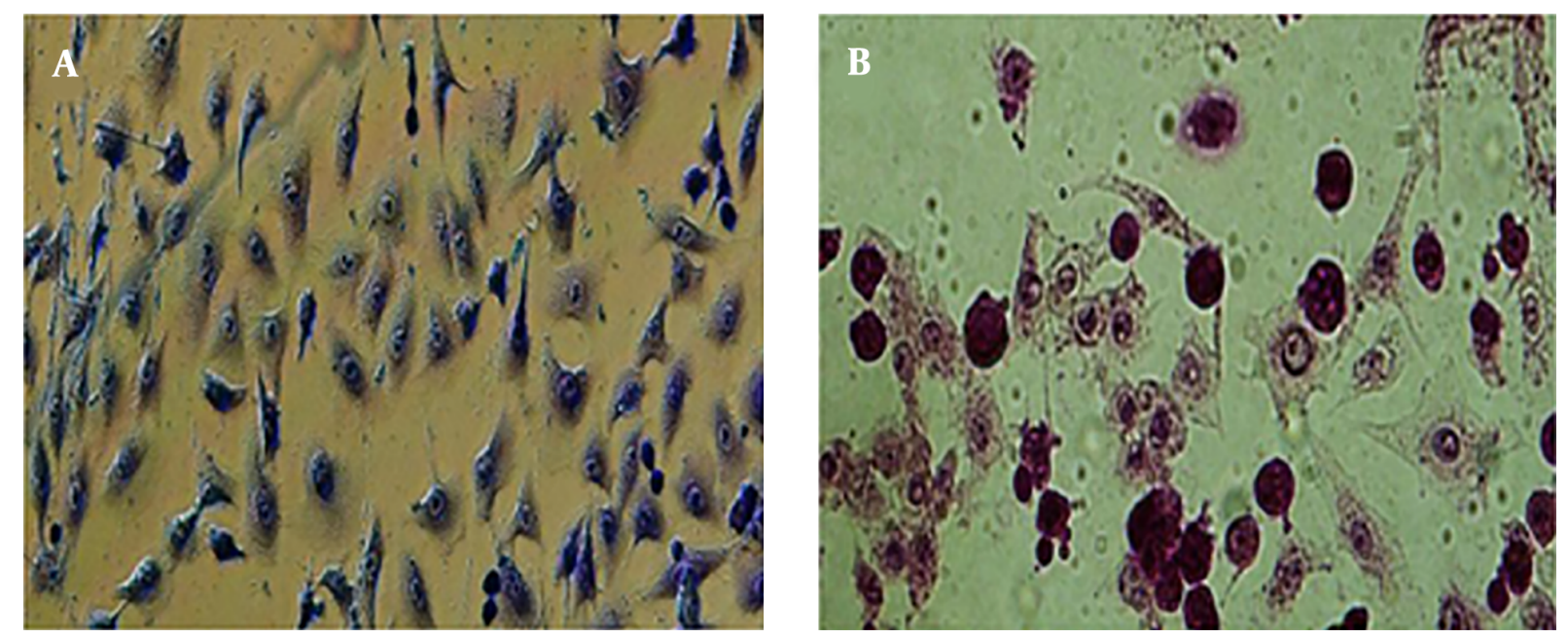

Figure 1. Morphological observation of A549 cells treated with a $2 \mu \mathrm{M}$ concentration of flubendazole using an inverted microscope one day after treatment. a) Control group. b). Apoptotic cells in the treated group. The images of cells were taken with an inverted microscope at 200X.

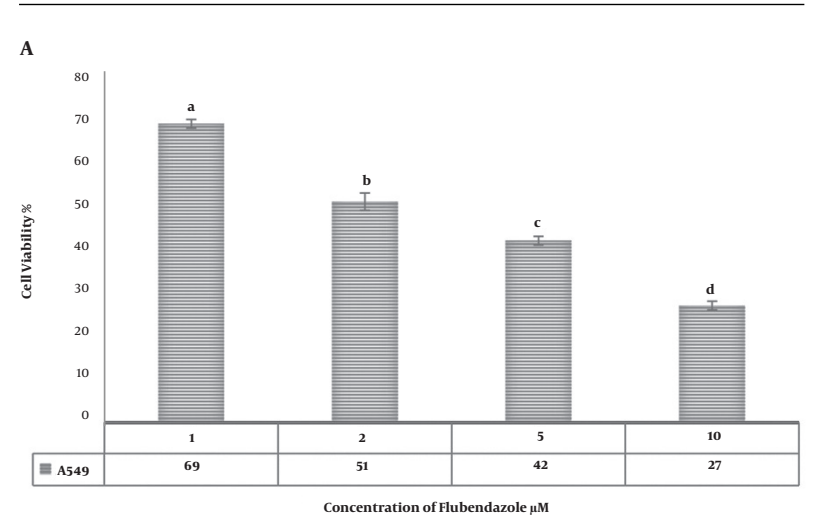

B

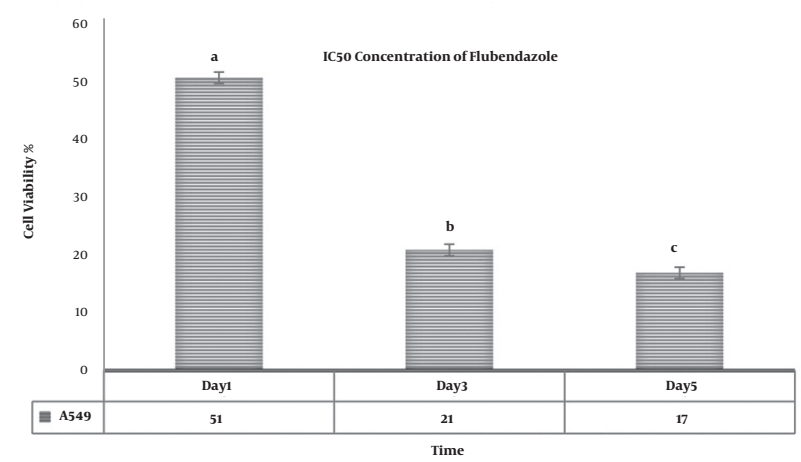

Figure 2. MTT assay for comparative cell viability of A549 cells treated with a) various concentrations of flubendazole one day after treatment; b) IC50 concentration of flubendazole one, two, and three days after treatment. Non-consecutive letters indicate significant differences at a confidence level of $\mathrm{P}<0.05$.

\section{Discussion}

The results of this study showed that flubendazole reduced the viability and proliferation of A549 human lung Jentashapir J Cell Mol Biol. 2020; 11(4):e108923. cancer cells in a dose and time-dependent manner. In other words, treatment of A549 cells with different doses of flubendazole increased cell death in proportion to drug dose and treatment time. According to the MTT results, flubendazole significantly decreased viability and could induce cell death in A549 cells. Also, the results of qRT-PCR showed that flubendazole could decrease P62 and increase Beclin 1 (as autophagy markers) in A549 cells.

Other researchers have shown that benzimidazole family members can affect different cancer cells by different anti-proliferative and anticancer mechanisms, some of which are discussed here $(13,14)$.

As a benzimidazole family member, albendazole can induce oxidative stress in cancer cells and induce a lethal effect. As Castro et al. (2016) showed in their study, albendazole has an antitumor effect by changing oxidative stress and inducing cell DNA damage to reduce the viability of MCF-7 line cells and prevent their colony formation. They also reported an increase in intracellular Reactive Oxygen Species (ROS) following treatment with albendazole and concluded that albendazole induces oxidative stress, degenerates DNA, increases apoptosis, and induces cell death (15). Thus, it can be a promising signal molecule to produce a new antitumor drug.

Another mechanism by which benzimidazole can exert its lethal effect is the inhibition of tubulin polymerization and cell proliferation $(16,17)$. In a study, Chu et al. (2009) evaluated the benzimidazole effect on human ovarian cancer cells (1AOPTX22 line), which were resistant to paclitaxel. They announced that benzimidazole inhibited human ovarian cancer cell proliferation via the inhi- 

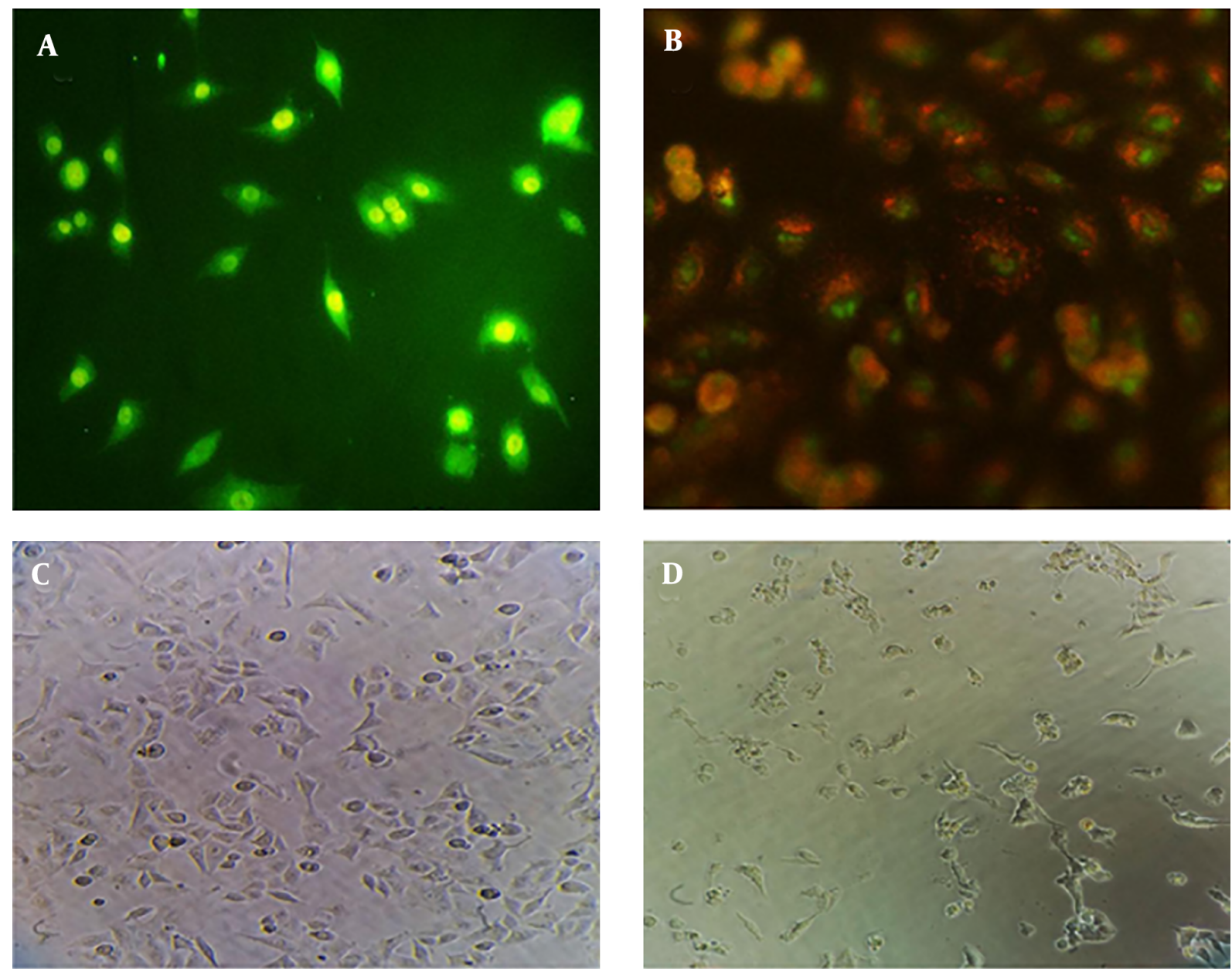

Figure 3. Morphological observation and acridine orange/ethidium bromide staining for checking apoptosis of A549 cells treated with IC50 concentration of flubendazole one day after treatment. a) Acridine orange/ethidium bromide staining for control cells; b) Acridine orange/ethidium bromide staining for treated cells. Here, orange-stained cells confirm cell apoptosis and green-stained cells indicate live cells in the control group. c) Morphological observation of control cells; b) Morphological observation of treated cells. The images of cells were taken with a fluorescence microscope at 100X (a and b) and an inverted microscope at 200X (c and d).

bition of tubulin polymerization $(18,19)$. Also, albendazole can stop mitosis in cancer cells. Zhang et al. evaluated the albendazole effect on human stomach cancer cells and expressed that albendazole has an anticancer effect by deforming microtubules and inhibiting mitosis. Also, albendazole induced apoptosis in these cells by inhibiting cyclin beta. Microtubules play a pivotal role in the cell cycle. Cancer experts have produced microtubular inhibitors that can prevent uncontrolled cell division, as observed in cancer. Apoptosis or cell programmed death is also a body standard mechanism to eliminate abnormal and unwanted cells and prevent tissues from becoming cancerous. Apoptotic activator drugs in the body seem to be good options for cancer therapy $(20,21)$. Patel et al. (2011) examined and compared the benzimidazole effect alone and in combination with radiotherapy on the viability of MM and SCLC colonic cell lines and showed that benzimidazole sensitizes these cell lines to radiation and its combination with radiation can be a potent treatment for MM and SCLC metastasis into the brain (18).

Flubendazole is another anthelminthic drug belonging to the benzimidazole family with anticancer effects by inhibiting microtubules function $(11,22)$. In a study, Michalis et al. (2015) examined the flubendazole effect on several cell lines of neuroblastoma cancer and introduced it as a potential anti-neuroblastoma compound. They announced that flubendazole could induce apoptosis mediated by the P53 protein and, in comparison with other anticancer drugs, is a potential treatment for neuroblastoma, including cells unreactive to therapy (23). 
IC50 Concentration

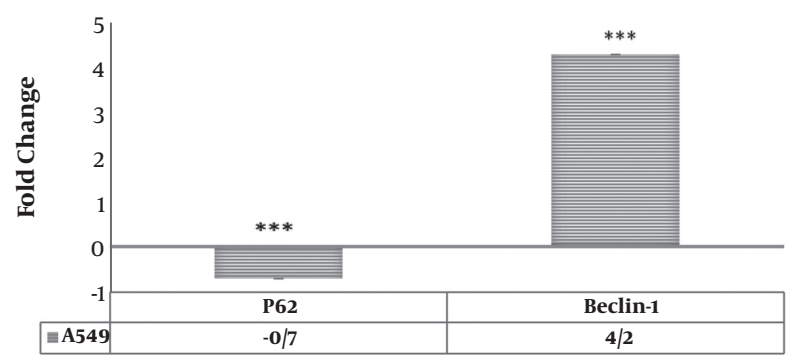

Figure 4. Gene expression changes in A549 cells treated with IC50 concentration of flubendazole after one day. Increased expression of the Beclin 1 gene and decreased expression of the P62 gene were observed in A549 cells treated with IC50 concentration of flubendazole. Also, differences in the expression of genes between treated and control cells have been shown by stars (***: P $<0.001)$. GAPDH was used as an internal modulator $(\mathrm{n}=3)$.

In 2015, Hou et al. investigated the effect of flubendazole on breast CS-like cells and demonstrated that flubendazole blocked breast cancer cell proliferation in a timeand dose-dependent manner and inhibited tumor growth in xenograft models through inhibiting tubulin polymerization. They showed the significant effect of flubendazole on inhibiting breast cells, indicating a new potential of flubendazole in breast cancer therapy (7).

Canova et al. in 2018 considered the cytotoxic effects of flubendazole on malignant melanoma in vitro on BOWES, RPMI-7951, and A-375 cell lines. Their results indicated that flubendazole inhibited cell proliferation and growth in three cell lines and changed cellular morphology. Also, they demonstrated that flubendazole disrupted the microtubule structure and arrested the cell cycle (12). In this regard, Tao et al. in 2019 studied the function of flubendazole in Esophageal Squamous Cell Carcinoma (ESCC). Their results indicated that flubendazole, as a novel NF- $\kappa \mathrm{B}$ inhibitor, could block the activation of $\mathrm{I} \kappa \mathrm{B} \alpha$ kinases and decrease the phosphorylation of NF- $\kappa$ B in ESCC cells. They also showed that flubendazole inhibited cell survival and increased cell apoptosis in EC9706 and TE1 cells. They announced that flubendazole had a synergistic effect with doxorubicin on ESCC cells (11).

\subsection{Conclusion}

Overall, the time and dose-dependent A549 cell viability and proliferation reduction following treatment with different doses of flubendazole in the present study show that flubendazole has a lethal effect on these cells. Thus, this study confirms the former findings of other researchers that flubendazole can be a promising therapy option for cancer treatment; however, more studies are needed in the future.

\section{Acknowledgments}

This work was supported financially from the research grants of authors (grant number: 1399). We acknowledge the Vice-President of Research and Technology of the Shahid Chamran University of Ahvaz.

\section{Footnotes}

Authors' Contribution: Hoveizi Elham contributed to the conception and design of all experimental work and carried out experiments. Fakharzade Fatemeh contributed to data and statistical analysis and manuscript writing. All authors read and approved the final manuscript.

Conflict of Interests: No conflict or competing financial interests exist.

Ethical Approval: This study is an experimental study on lung A549 cancer cell line conducted at Cell and Developmental Lab of the Biology Department, Faculty of Sciences, Shahid Chamran University (ethical code: EE.97.243.93365/scu.ac.ir).

Funding/Support: This study was funded and supported by the Shahid Chamran University of Ahvaz.

\section{References}

1. D'Arcy MS. Cell death: a review of the major forms of apoptosis, necrosis and autophagy. Cell Biol Int. 2019;43(6):582-92. doi: 10.1002/cbin.11137. [PubMed: 30958602].

2. Al Sawaftah NM, Husseini GA. Ultrasound-Mediated Drug Delivery in Cancer Therapy: A Review. J Nanosci Nanotechnol. 2020;20(12):7211-30. doi: 10.1166/jnn.2020.18877. [PubMed: 32711586].

3. Dudzinska M, Szczyrek M, Wojas-Krawczyk K, Swirska J, Chmielewska I, Zwolak A. Endocrine Adverse Events of Nivolumab in Non-Small Cell Lung Cancer Patients-Literature Review. Cancers (Basel). 2020;12(8). doi: 10.3390/cancers12082314. [PubMed: 32824462]. [PubMed Central: PMC7466155].

4. Rudolf K, Rudolf E. An analysis of mitotic catastrophe induced cell responses in melanoma cells exposed to flubendazole. Toxicol In Vitro. 2020;68:104930. doi: 10.1016/j.tiv.2020.104930. [PubMed: 32652169].

5. Oh E, Kim YJ, An H, Sung D, Cho TM, Farrand L, et al. Flubendazole elicits anti-metastatic effects in triple-negative breast cancer via STAT3 inhibition. Int J Cancer. 2018;143(8):1978-93. doi: 10.1002/ijc.31585. [PubMed: 29744876].

6. Nath J, Paul R, Ghosh SK, Paul J, Singha B, Debnath N. Drug repurposing and relabeling for cancer therapy: Emerging benzimidazole antihelminthics with potent anticancer effects. Life Sci. 2020;258:118189. doi: 10.1016/j.lfs.2020.118189. [PubMed: 32781060].

7. Hou ZJ, Luo X, Zhang W, Peng F, Cui B, Wu SJ, et al. Flubendazole, FDA-approved anthelmintic, targets breast cancer stem-like cells. Oncotarget. 2015;6(8):6326-40. doi: 10.18632/oncotarget.3436. [PubMed: 25811972]. [PubMed Central: PMC4467440].

8. Garcia CS, Menti C, Lambert AP, Barcellos T, Moura S, Calloni C, et al. Pharmacological perspectives from Brazilian Salvia officinalis (Lamiaceae): antioxidant, and antitumor in mammalian cells. An Acad Bras Cienc. 2016;88(1):281-92. doi: 10.1590/0001-3765201520150344. [PubMed: 26839997]. 
9. Zhen Y, Zhao R, Wang M, Jiang X, Gao F, Fu L, et al. Flubendazole elicits anti-cancer effects via targeting EVA1A-modulated autophagy and apoptosis in Triple-negative Breast Cancer. Theranostics. 2020;10(18):8080-97. doi: 10.7150/thno.43473. [PubMed: 32724459]. [PubMed Central: PMC7381743]

10. Lin S, Yang L, Yao Y, Xu L, Xiang Y, Zhao H, et al. Flubendazole demonstrates valid antitumor effects by inhibiting STAT3 and activating autophagy. J Exp Clin Cancer Res. 2019;38(1):293. doi: 10.1186/s13046-0191303-z. [PubMed: 31287013]. [PubMed Central: PMC6615228].

11. Tao J, Zhao H, Xie X, Luo M, Gao Z, Sun H, et al. The anthelmintic drug flubendazole induces cell apoptosis and inhibits NF-kappaB signaling in esophageal squamous cell carcinoma. Onco Targets Ther. 2019;12:471-8. doi:10.2147/OTT.S193206. [PubMed:30666126]. [PubMed Central: PMC6331185].

12. Canova K, Rozkydalova L, Vokurkova D, Rudolf E. Flubendazole induces mitotic catastrophe and apoptosis in melanoma cells. Toxicol In Vitro. 2018;46:313-22. doi: 10.1016/j.tiv.2017.10.025. [PubMed: 29107018].

13. Akhtar S, Abbas M, Naeem K, Faheem M, Nadeem H, Mehmood A. Benzimidazole Derivative Ameliorates Opioid-mediated Tolerance during Anticancer-Induced Neuropathic Pain in mice. Anticancer Agents Med Chem. 2020. doi: 10.2174/1871520620999200818155031. [PubMed: 32819235].

14. Munoz-Patino N, Sanchez-Eguia BN, Araiza-Olivera D, Flores-Alamo M, Hernandez-Ortega S, Martinez-Otero D, et al. Synthesis, structure, and biological activity of bis(benzimidazole)amino thio- and selenoether nickel complexes. J Inorg Biochem. 2020;211:111198. doi: 10.1016/j.jinorgbio.2020.111198. [PubMed: 32801056].

15. Castro LS, Kviecinski MR, Ourique F, Parisotto EB, Grinevicius VM, Correia JF, et al. Albendazole as a promising molecule for tumor control. Redox Biol. 2016;10:90-9. doi: 10.1016/j.redox.2016.09.013. [PubMed: 27710854]. [PubMed Central: PMC5053114].

16. Driscoll M, Dean E, Reilly E, Bergholz E, Chalfie M. Genetic and molecular analysis of a Caenorhabditis elegans beta-tubulin that conveys benzimidazole sensitivity. J Cell Biol. 1989;109(6 Pt 1):29933003. doi: 10.1083/jcb.109.6.2993. [PubMed: 2592410]. [PubMed Central: PMC2115974].
17. Chang WL, Chang CS, Chiang PC, Ho YF, Liu JF, Chang KW, et al. 2-Phenyl-5-(pyrrolidin-1-yl)-1-(3,4,5-trimethoxybenzyl)-1Hbenzimidazole, a benzimidazole derivative, inhibits growth of human prostate cancer cells by affecting tubulin and cJun N-terminal kinase. Br J Pharmacol. 2010;160(7):1677-89. doi 10.1111/j.1476-5381.2010.00832.x. [PubMed: 20649571]. [PubMed Central: PMC2936840].

18. Patel K, Doudican NA, Schiff PB, Orlow SJ. Albendazole sensitizes cancer cells to ionizing radiation. Radiat Oncol. 2011;6:160. doi: 10.1186/1748-717X-6-160. [PubMed: 22094106]. [PubMed Central: PMC3231941]

19. Chao G, Xiaobo S, Chenglin C, Yinsheng D, Yuepu P, Pinghua L. A cellular automaton simulation of the degradation of porous polylactide scaffold: I. Effect of porosity. Materials Science and Engineering: $C$. 2009;29(6):1950-8. doi:10.1016/j.msec.2009.03.003.

20. Zhang X, Zhao J, Gao X, Pei D, Gao C. Anthelmintic drug albendazole arrests human gastric cancer cells at the mitotic phase and induces apoptosis. Exp Ther Med. 2017;13(2):595-603. doi 10.3892/etm.2016.3992. [PubMed: 28352336]. [PubMed Central: PMC5348670].

21. Wang LJ, Liou LR, Shi YJ, Chiou JT, Lee YC, Huang CH, et al. AlbendazoleInduced SIRT3 Upregulation Protects Human Leukemia K562 Cells from the Cytotoxicity of MCL1 Suppression. Int J Mol Sci. 2020;21(11). doi: 10.3390/ijms21113907. [PubMed: 32486166]. [PubMed Central: PMC7312678]

22. Li Y, Acharya G, Elahy M, Xin H, Khachigian LM. The anthelmintic flubendazole blocks human melanoma growth and metastasis and suppresses programmed cell death protein-1 and myeloid-derived suppressor cell accumulation. Cancer Lett. 2019;459:268-76. doi 10.1016/j.canlet.2019.05.026. [PubMed: 31128215].

23. Michaelis M, Agha B, Rothweiler F, Loschmann N, Voges Y, Mit telbronn $\mathrm{M}$, et al. Identification of flubendazole as potential antineuroblastoma compound in a large cell line screen. Sci Rep. 2015;5:8202. doi: 10.1038/srep08202. [PubMed: 25644037]. [PubMed Central: PMC4314641]. 